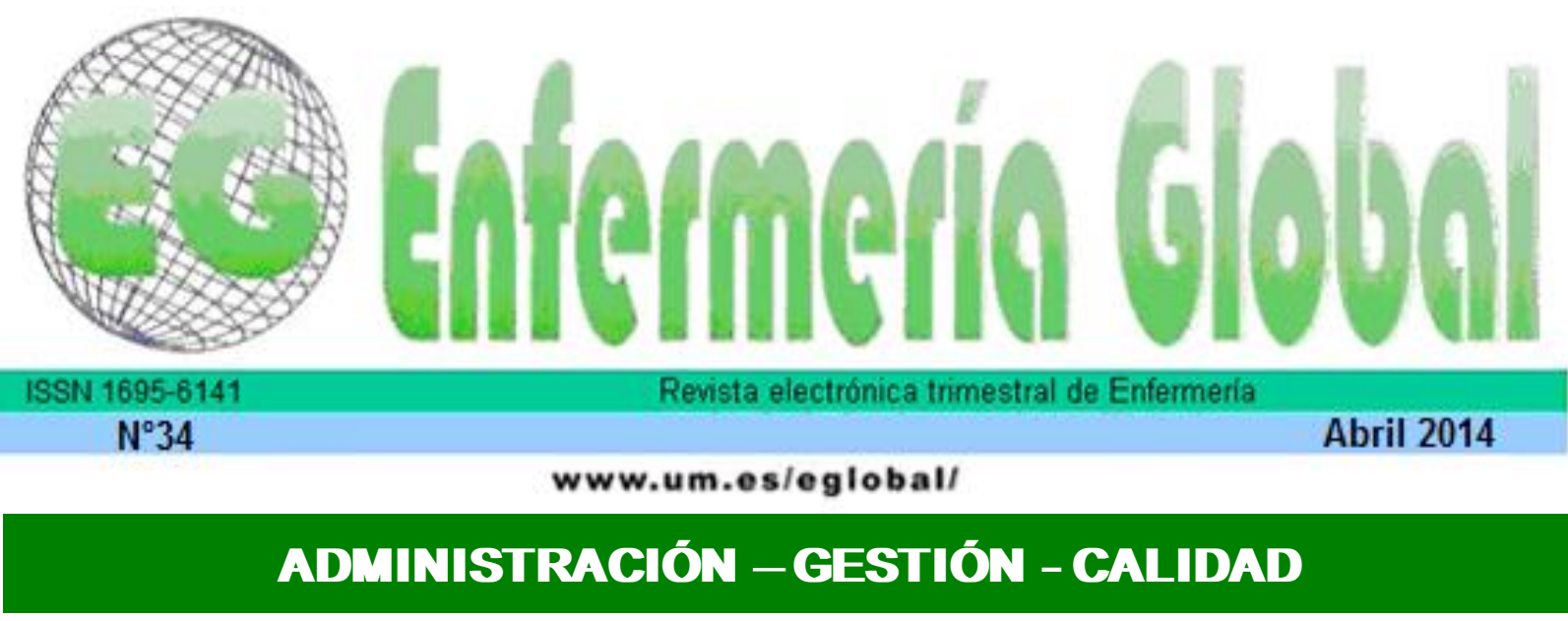

\title{
Eventos adversos: instrumento de gerencia de la asistencia para la seguridad del paciente en el servicio de urgencias
}

Incidentes: instrumento de gerenciamento da assistência para a segurança do paciente em pronto socorro

Incidents: instrument management assistance for patient safety in emergency room

\section{*Paranaguá, Thatianny Tanferri de Brito **Braga, Queren de Pádua ***Bezerra, Ana Lúcia Queiroz ${ }^{* * * *}$ Bauer de Camargo e Silva, Ana Elisa ${ }^{* * * * *}$ Azevedo Filho, Francino Machado de ${ }^{* * * * *}$ Gomes de Sousa, Maiana Regina}

*Máster en Enfermería. Doctoranda del Programa de Pos-Graduación de la Universidad Federal de Goiás. E-mail: ttb.paranagua@gmail.com ** Enfermera. Hospital de Urgencias de Aparecida de Goiânia. *** Doctora en Enfermería. Profesora Asociada de la Facultad de Enfermería de la Universidad Federal de Goiás **** Profesor Adjunto de la Facultad de Enfermería de la Universidad Federal de Goiás ****Enfermero. Profesor de la Universidad Estadual de Goiás. ${ }^{* * * * * E n f e r m e r a . ~}$ Alumna de Máster del Programa de Pos-Graduación de la Facultad de Enfermería de la Universidad Federal de Goiás. Brasil.

Palabras clave: Evaluación de procesos y resultados (atención de salud); garantía de calidad de atención de salud; servicios de urgencia en hospital; enfermería.

Palavras chave: Avaliação de processos e resultados (cuidados de saúde); garantia da qualidade dos cuidados de saúde; serviço hospitalar de emergência; enfermagem.

Keywords: Outcome and process assessment (health care); quality assurance, health care; emergency service, hospital; nursing.

\section{RESUMEN}

Este estudio objetivó caracterizar los incidentes derivados de los cuidados ocurridos en el servicio de urgencias de un hospital universitario perteneciente a la Red de Hospitales Centinela. Estudio descriptivo, retrospectivo, que analizó las notas registradas entre septiembre de 2007 hasta marzo de 2010, en los libros de anotaciones de enfermería. Los datos fueron recolectados entre enero y marzo de 2010, estructurados en hoja de cálculo y analizados descriptivamente, presentando las frecuencias absolutas y relativas. Se identificó el registro de 75 incidentes que fueron relacionados con la 
organización del servicio o del cuidado. Se constató el mayor índice de errores de medicación, omisión de cuidado y fallos en las anotaciones y/o registros en la hoja de evolución del paciente, destacando el óbito como la consecuencia más grave. Los resultados señalan la necesidad de estimular la construcción y uso de instrumentos de notificación e incentivar la notificación completa de los incidentes, ya que estos son herramientas fundamentales de calidad y pueden dirigir la gestión de los servicios frente a los indicadores que deben ser mejorados en el lugar de trabajo para así proporcionar atención segura y de calidad.

\section{RESUMO}

O estudo objetivou caracterizar os incidentes advindos do cuidado à saúde, ocorridos no Pronto Socorro de um hospital universitário que pertence à Rede de Hospitais Sentinela. Estudo descritivo, retrospectivo que analisou as anotações realizadas entre setembro de 2007 e março de 2010, nos livros de intercorrência da unidade. Os dados foram coletados entre janeiro e março de 2010, estruturados em planilha eletrônica e analisados descritivamente, apresentando as frequências absoluta e relativa. Identificou-se o registro de 75 incidentes que foram relacionados à organização do serviço ou ao cuidado. Constatou-se o maior índice de erro de medicação, omissão de cuidado e falha nas anotações e/ou registros na folha de evolução do paciente, destacando o óbito como a consequência mais grave. Os resultados apontam a necessidade de estimular a construção e uso de instrumentos de notificação e incentivar a notificação completa dos incidentes, já que constituem ferramentas fundamentais da qualidade e podem direcionar a gestão dos serviços frente a indicadores que devem ser melhorados no ambiente de trabalho para, assim, oferecer uma assistência segura e de qualidade.

\section{ABSTRACT}

This study aims to characterize the incidents arising of the health care, occurred in the emergency department of a university hospital of the sentinel hospitals network. Descriptive and retrospective study which examined notes that were made between September 2007 and March 2010, recorded in the books of intercurrence of this unity. Data were collected between January and March, 2010, structured in worksheets and analyzed descriptively, presenting the relative and absolute frequencies. Were identified the register of 75 incidents that were related to organization in the service or to the care. Were found more errors indices in medication, care omission and in notes and/or register in the sheet of evolution of the patient, highlighting the death as more serious consequence. The results points to the need of stimulate the construction and use of instruments of notification and incentive to the full notification of the incidents, because it constitute fundamental tool of quality and can orientate the service management face to indicators that should be improved in the environment of work to, thereby provide safe security and with quality.

\section{INTRODUCCIÓN}

La seguridad del paciente es fundamental para que los hospitales cumplan su papel social, ofreciendo una asistencia sanitaria de calidad con el mínimo de riesgos al paciente. En este sentido, se ha dado una atención especial a la ocurrencia de incidentes, indicadores de calidad en salud, definidos como eventos indeseables derivados del cuidado, que comprometen la seguridad del paciente ${ }^{(1)}$. El incidente que resulta en algún tipo de daño al paciente es clasificado como evento adverso ${ }^{(2)}$.

O The Australian Quality Health Care Study (QAHCS), realizado en 1992, definió evento adverso como lesión no intencional que resulta en incapacidad temporal o permanente $y / 0$ en prolongación del tiempo de permanencia o muerte, como consecuencia del cuidado prestado ${ }^{(3)}$.

El aumento de ocurrencia es señalado como uno de los factores que influyen directamente en la calidad de los servicios sanitarios, lo que ha provocado debates en el ámbito internacional, considerando que los incidentes pueden resultar, además de 
en tragedia para los pacientes y/o familiares, en aumento de los costos de la asistencia sanitaria ${ }^{(4)}$.

Estudios señalan que entre las condiciones facilitadoras de la ocurrencia de los incidentes están el fracaso de comunicación entre los profesionales y entre estos y los pacientes, la fragmentación de la responsabilidad por la asistencia, la discontinuidad en la atención, lo inadecuado de los sistemas de información, la insuficiente incorporación de protocolos clínicos elaborados por medio de bases científicas sólidas y el fallo en los mecanismos de registro y notificación de estos eventos ${ }^{(5)}$.

Se sabe que el incidente se debe a múltiples factores, tales como procedimientos quirúrgicos, utilización de medicamentos, procedimientos médicos, tratamiento no medicamentoso, demora o equivocación en el diagnóstico, siendo más frecuentes y graves en el ámbito hospitalario ${ }^{(2)}$.

Dentro de este contexto, la unidad de Urgencias se constituye en área de alto riesgo para la ocurrencia de diversos tipos de incidentes debido a la rotatividad y dinámica de la atención, al absorber gran demanda de pacientes con diversos grados de gravedad, además de convivir con deficiencia cuantitativa y cualitativa de personal y recursos materiales que resultan en sobrecarga de trabajo y estrés profesional y ambiental $^{(5,6)}$.

En Estados Unidos, se estima que 100.000 personas mueren en hospitales cada año víctimas de incidentes, con un gasto entre 17 y 29 billones de dólares ${ }^{(3)}$.

En Brasil, estudios comparando las proporciones de incidentes atribuidos a negligencia en diferentes sectores hospitalarios revelan que Urgencias fue el servicio de mayor concentración, con una ocurrencia del $50 \%$ al $70 \%$ de los eventos detectados $^{(7)}$.

Sin embargo, se estima que en torno al $60 \%$ de los incidentes relacionados con la asistencia sanitaria se consideran evitables ${ }^{(4)}$. Considerando que el incidente está relacionado con un fallo humano, su ocurrencia está asociada, frecuentemente, a la vergüenza, castigos y pérdida de prestigio por parte de los profesionales y debido a la falta de orientación sobre la conducta a tomar frente al evento la tendencia es ocultarlo, especialmente en los casos en que resulten daños, perdiéndose una gran oportunidad de aprendizaje ${ }^{(8,9)}$.

Esto se debe al hecho de que el abordaje de los errores en el sistema sanitario se hace de forma individual, considerando el error un acto inseguro cometido por personas con formación deficiente o falta de atención ${ }^{(8)}$.

Esta realidad refleja la necesidad de establecer acciones de control y prevención de los incidentes, estimular la notificación y eliminar los sentimientos de pena por parte de los profesionales involucrados y reconocer que la organización del modo de trabajo en el sistema sanitario también puede influir en la ocurrencia del incidente, asumiendo una proporción sistémica.

La dificultad en la implementación de la Política Nacional de Salud torna los servicios de Urgencias en centro del sistema y en el principal agente de detección. Reconociendo que los incidentes pueden ser minimizados y permiten medir la calidad de la asistencia sanitaria y de enfermería en ese servicio, el presente estudio tiene relevancia para permitir el desarrollo de estrategias que prevengan tales ocurrencias, 
garanticen la seguridad del paciente y, por consiguiente, aumenten la calidad del servicio.

Frente a esto, el presente estudio tiene como objetivo caracterizar los incidentes registrados en los libros de interocurrencias de Urgencias de un hospital universitario de la Región Centro-Oeste.

\section{MÉTODO}

Se trata de estudio descriptivo, retrospectivo, desarrollado en Urgencias de un Hospital Universitario de la Región Centro-Oeste, perteneciente a la Red Hospitales Centinela, creada en 2004 por la Agencia Nacional de Vigilancia Sanitaria. Esta red fue creada para vigilar eventos adversos y quejas técnicas derivadas de la asistencia sanitaria para planificar acciones preventivas $^{(6)}$.

Urgencias, campo de este estudio, cuenta con capacidad física para 58 camas y atiende una media de 1.654 pacientes/mes, siendo 19.848 pacientes/año.

Se utilizó como fuente de datos, el libro de interocurrencias de la unidad, que es de acceso exclusivo y voluntario del equipo de enfermería y tiene la finalidad de registrar cualquier interocurrencia relacionada con el servicio y los pacientes.

La población del estudio constó de los incidentes ocurridos en el período de 19 de septiembre de 2007 a 04 de marzo de 2010, registrados por los enfermeros, en los libros de intercocurrencias.

La colecta de datos se realizó en los meses de enero a marzo de 2010, utilizando un instrumento estructurado, conteniendo cuestiones sobre fecha, horario y tipo de incidente, consecuencias para el paciente, profesionales involucrados e intervenciones realizadas por los profesionales inmediatamente después de la ocurrencia del evento. El instrumento fue sometido a la evaluación de cinco especialistas, investigadores del área de eventos adversos y docentes de las disciplinas de enfermería fundamental y administración de servicios sanitarios y enfermería. A continuación se realizó el test piloto para la adecuación y mejora de la calidad del instrumento en cuanto a claridad y objetividad.

Los datos se transcribieron íntegramente en documento de Microsoft Word para formar el corpus de análisis, atribuyéndose la letra " $R$ " seguida de número cardinal para cada registro, de acuerdo con el orden de recogida. Para preservar el anonimato de pacientes y profesionales involucrados en el evento, sus nombres fueron retirados, sin perjudicar la integridad de los registros. En seguida, los datos se organizaron en planillas electrónicas de Microsoft Excel, versión 2007, analizados estadísticamente y presentadas frecuencias absoluta y relativa.

Estudio apreciado y aprobado por el Comité de Ética de la institución, bajo Protocolo $N^{\circ}$ 064/2008. Se siguieron todos los aspectos éticos, conforme la Resolución del Consejo Nacional de Salud № 196/96.

\section{RESULTADOS}

Se constató el registro de 75 incidentes que abarcaron aspectos institucionales y asistenciales, estimando prevalencia de 1,5\%. 
La tabla 1 presenta los incidentes relacionados con la organización de los servicios.

Tabla 1 - Distribución de los incidentes relacionados con la organización del servicio. Goiânia (GO), 2007-2010.

\begin{tabular}{lcc}
\hline EVENTOS ADVERSOS & $\mathbf{n}$ & $\%$ \\
\hline Fallos en las anotaciones & & \\
Ausencia de anotaciones y/o registro incorrecto & 17 & 58,6 \\
Comprobación de medicación inadecuada & 4 & 13,8 \\
Previsión y provisión de materiales & 4 & 13,8 \\
Evasión del paciente & 4 & 13,8 \\
\hline Total & 29 & 100,0 \\
\hline
\end{tabular}

De entre los 29 incidentes relacionados con la organización del servicio, el más frecuente está relacionado con los errores en las anotaciones, caracterizadas como ausencia de anotaciones y/o registro incorrecto de signos vitales y balance hídrico, comprobación de medicación inadecuada y dificultad para realizar la lectura de medicamentos prescritos en la historia clínica: "Paciente de la cama 2 está con el medicamento alopurinol pautado en diferentes horarios, mas no hay esta medicación en la farmacia y el paciente tampoco la tiene" (R22), "Paciente nefrópata con prescripción médica para control de cantidad de orina, el 'profesional' relató dos veces en el recipiente, sin embargo, el paciente está usando sonda vesical permanente" (R30).

También se identificó $13,8 \%$ de incidentes referentes a previsión y provisión de materiales, tales como el abastecimiento inadecuado del carro de emergencia, la deficiencia en la reposición y en el mantenimiento, tanto de materiales como de equipos, en el inicio del servicio y tras la atención.

Fallos en la previsión y provisión de estos materiales acarrearon graves incidentes al paciente, conforme evidenciado en los registros: "Estamos con dos pacientes para ser entubados, mas sólo tenemos un ventilador - los otros están averiados. UCI y Clínicas no tienen equipamiento disponible para préstamo" (R2), "Paciente hizo Parada Cardiorrespiratoria, fue reanimada durante más de 30 min, mecánica y químicamente, sin éxito. En aquel momento nuestros ambus (todos) estaban en desinfección química y habíamos cogido prestado un ambu en la UCl quirúrgica que no funcionaba! Los tubos endotraqueales estaban rasgados, rotos (tapado con esparadrapo), una de las salídas de vacío no funcionó en ese momento. Todos se quejaron de la falta de material, de los daños y de las condiciones de trabajo" (R7).

Otro evento relacionado con la organización del servicio fue la fuga del paciente de la unidad de ingreso que correspondió a $13,8 \%$ de los incidentes: "Paciente se fugó alrededor de las $13 \mathrm{~h}$ y $30 \mathrm{~min}$, retornando a las $15 \mathrm{~h}$ y 50min por el cuerpo de bomberos que lo encontró sin ropa y embriagado"(R11).

En cuanto a las consecuencias, se comprobó que en $26(89,7 \%)$ incidentes no había anotación sobre las conductas en relación al paciente, familia y profesional involucrado; dos $(6,9 \%)$ informaron la ausencia de daño y uno $(3,5 \%)$ resultó en óbito.

Los incidentes relacionados con el cuidado fueron identificados en 46 registros, conforme tabla 2. 
Tabla 2 - Distribución de los incidentes relacionados con el cuidado. Goiânia (GO), 2007-2010.

\begin{tabular}{lcc}
\hline EVENTOS ADVERSOS & $\mathbf{n}$ & $\%$ \\
\hline Omisión del cuidado & 18 & 39,1 \\
Error de medicación & & \\
Omisión de la dosis & 7 & 15,2 \\
Cambio de paciente & 7 & 15,2 \\
Falta de medicamento & 4 & 8,7 \\
Administración de medicamento no prescrito & 1 & 2,2 \\
Falta de humanización & 4 & 8,7 \\
Infección hospitalaria & 2 & 4,4 \\
Caída & 2 & 4,4 \\
Fallo en el procedimiento/técnica & 1 & 2,2 \\
\hline Total & 46 & 100,0 \\
\hline
\end{tabular}

Los incidentes relacionados con la omisión del cuidado, como la no realización de curativos y ausencia de la verificación de signos vitals, de glicemia, de balance hídrico, entre otros, ocurrieron con mayor frecuencia: "He oído del paciente y de su acompañante que la cura de la tarde no se hizo porque no había nadie para ello" (R14), "Pedi una placa de hidrocoloide en la farmacia de Urgencias a las 08h30min. Hasta ahora, 12h 00min, no fue entregada. Hubo discontinuidad del tratamiento" (R26,) "Hoy, 12/01/2010 período vespertino, la Sra [...] fue subiendo en los cuidados de la sala 01, 02 y 06 (totalizando 15 pacientes), cuidados que incluyen: signos vitales, HGT, curas, débito de diuresis y controles en general, sin embargo, esta misma "profesional" no hizo nada. Todos los signos vitales no fueron verificados, y sí solamente copiados" (R30).

En cuanto a los errores de medicación, se evidenció $41,3 \%$ de eventos relacionados con las omisiones de dosis, cambios de pacientes, falta de medicamentos y administración de medicamentos no prescritos: "Equipo médico reclamó por la no administración de dipirona SOS al paciente que tenía $38^{\circ} \mathrm{C}$ de temperatura a las 20 horas del mismo servicio" (R15), "Por segunda vez hubo cambio de sueros (rótulos de pacientes en observación durante el nocturno). $1^{\circ}$ vez: se cambió el suero de los pacientes y por suerte tenían la misma composición e goteo. $2^{\circ}$ vez: se cambió el suero de los pacientes también de la misma composición y goteo, gracias a Dios" (R3), "Hoy hubo un cambio de pacientes en el momento de administrar oxacilina. El medicamento era del paciente $X$ y se inyectó al paciente $Y$ " (R23).

También se identificó $8,7 \%$ de incidentes relacionados con la humanización: "Hoy tuvimos dos urgencias que precisamos de evaluación del otorrino, una fue niño con otorragia y una mujer con una cucaracha en el oido. Hablé con el residente que me dijo que no tenía obligación de verla aqui y que no era realmente una emergencia. El niño no fue atendido y la mujer estuvo desde las $6 \mathrm{~h}$ de la mañana hasta las $16 \mathrm{~h}$ sin atención" (R4), "Paciente con hematemesis es llevada para reanimación. No fue encontrado acceso venoso periférico. El de guardia fue avisado, mas no compareció en la sala de reanimación. Paciente evolucionó a PCR. A las 18h10min: óbito" (R13).

Otro evento levantado fue la ocurrencia de infecciones asociadas al cuidado: "Paciente presenta infección por Stafilococus aureus multirresistente según resultado de laboratorio". (R21) "El paciente [...] que se encuentra ingresado está colonizado con la bacteria Klebsiella pneumoniae" (R9). 
También en esta categoría, 4,4\% de los incidentes se relacionó con las caídas: "Paciente sufrió caída de la hamaca lo que nos lleva a pensar una vez más en quitar las hamacas y poltronas de Urgencias, intentando dar un poco de confort a los pacientes" (R17).

También fue identificado incidente relacionado con el fallo en el procedimiento/técnica, correspondiendo a 2,2\% de los eventos: "En el período vespertino, durante la administración de insulina según HGT, la funcionaria utilizó la mesma jeringa del paciente $A$ en el paciente B" (R19).

No se encontraron registros sobre consecuencias al paciente en $31(67,4 \%)$ incidentes. $9(19,6 \%)$ resultaron en daño, requiriendo seguimiento y/o prolongando el tiempo de ingreso, seguido de $5(10,9 \%)$ que no causaron daño aparente y un $(2,2 \%)$ resultó en óbito.

Los registros sobre conductas adoptadas tras el incidente tampoco fueron identificados.

\section{DISCUSIÓN}

El método de estudio presenta limitaciones en cuanto al número de incidentes identificados, ya que el registro es voluntario y la fuente de datos utilizada no constituye instrumento propio para la notificación. Aunque el cuantitativo de incidentes pueda considerarse bajo en relación al intervalo de tiempo investigado, los resultados señalan inúmeros indicadores de asistencia sanitaria, frecuentemente encontrados en la literatura, pero que todavía son identificados rutinariamente en el ambiente de trabajo.

La estimación de $1,5 \%$ de incidentes constatados infiere la baja notificación y la ausencia de una cultura de seguridad.

La notificación de interocurrencias durante la asistencia sanitaria se considera una estrategia eficaz para gerenciar, disminuir y controlar la ocurrencia de los incidentes, pues ayuda a la planificación de acciones de formación continua y dirige las acciones de mejora de los servicios, además de permitir la evaluación de los resultados ${ }^{(10)}$.

Se acredita que la reciente publicación del Decreto № 529 de $1^{\circ}$ de abril de 2013, del Ministerio de la Salud de Brasil, que instituye el Programa Nacional de Seguridad, podrá contribuir a la producción, sistematización y difusión de conocimientos sobre los incidentes, además de fomentar la cultura de seguridad del paciente en las instituciones de formación y práctica sanitaria ${ }^{(11)}$.

Se identificaron 29 incidentes relacionados con los fallos en las anotaciones, en la previsión y provisión de materiales y en la fuga del paciente de la unidad de ingreso, que evidenciaron la necesidad de despertar en los profesionales el compromiso ético y la responsabilización por la práctica asistencial.

Estudio evaluativo de los registros de enfermería verificó que la comprobación de la prescripción médica estaba ausente en $1,9 \%$ de las historias clínicas y de entre los $98,1 \%$ de las historias con comprobación, solo $1,7 \%$ tenían la comprobación completa. El análisis cualitativo de las historias clínicas indicó que $26,7 \%$ de las anotaciones se consideraron malas; $64,6 \%$, regulares y solo $8,7 \%$, buenas. Ninguna anotación se evaluó como óptima ${ }^{(12)}$. 
El registro incompleto de las situaciones relacionadas con la asistencia prestada al paciente imposibilita su uso como instrumento de comunicación. Las informaciones prestadas en calquier documento deben ser claras, objetivas, frecuentes y completas a fin de posibilitar el seguimiento, la evaluación y la planificación continua de los cuidados $^{(13)}$.

Esta realidad se hace evidente en numerosos sectores del sector salud ${ }^{(12-14)}$. Se configura como un factor agravante, visto que las anotaciones de las historias clínicas o cualquier documento de registro de informaciones en los servicios de salud tienen valor significativo, siendo fuentes de investigación, instrumento de educación y documento legal ${ }^{(12)}$.

Se cree, por tanto, que la enfermería debe evaluar sistemáticamente su propia actuación, en el sentido de reducir problemas relacionados con las anotaciones, considerando la importancia de los registros para la mejora de la calidad de la asistencia al paciente ${ }^{(13)}$ y, también, para garantizar mayor visibilidad a la categoría profesional.

Para una asistencia segura y de calidad es favorable un adecuado dimensionamiento del personal, sin embargo, se configura acción fundamental, el suministro adecuado de recursos materiales. Las instituciones de salud deben adoptar una rutina de reposición y mantenimiento de materiales, pues la insuficiencia o fallo de funcionamiento pueden acarrear pérdida de tiempo para el equipo y también daños irreversibles al paciente ${ }^{(15)}$, como se constata en los registros de los enfermeros.

Verificar el funcionamiento del ventilador mecánico, del desfibrilador, del aspirador, del laringoscopio, del ambu y demás equipos de una unidad de salud es una acción simple frente a la magnitud de las consecuencias que la falta y el mantenimiento inadecuado de estos equipos pueden acarrear. Interocurrencias de alto grado de complejidad resultan en óbito del paciente, frecuentemente, en los servicios de Urgencias, lo que exige la perfecta adecuación de los procesos de gestión de materiales, además de equipo eficiente, con conocimiento científico y habilidad técnica ${ }^{(15)}$.

La participación de los enfermeros en el proceso de compra de materiales en los hospitales viene siendo cada vez más requerida, teniendo en cuenta su competencia para opinar en cuanto al tipo, a la cantidad y a la calidad de los materiales a ser adquiridos para su utilización en las salas de curas ${ }^{(16)}$. Informalmente, el enfermero siempre participó de este proceso, de forma no sistematizada y siempre paralelamente a otras tareas, evidenciando la importancia de planear y sistematizar la administración de recursos materiales en los servicios de salud.

En Brasil, la cuestión de la calidad de la sistematización de la asistencia sanitaria, principalmente de enfermería, se configura como uno de los problemas de planificación en salud, lo que dificulta la previsión de recursos materiales, así como el establecimiento de la relación entre oferta de servicios y demandas de salud. La identificación de estas dificultades es necesaria e imprescindible, pues posibilita la redirección de recursos y actividades a sectores más problemáticos o de mayor riesgo, lo que, ciertamente, resulta en reducción de los recursos gastados ${ }^{(16)}$.

Las tasas de evasión, otro incidente identificado, constituyen buenos indicadores para evaluar la superpoblación de las unidades de Emergencias. Comúnmente los pacientes se enfrentan a grandes colas, largos períodos de espera, pacientes y 
acompañantes preocupados, irritados y cansados. Algunos pacientes con enfermedades sin gravedad inmediata podrían ser atendidos en ambulatorios $u$ otros servicios, mientras que aquellos que realmente necesitan de atención urgente, acaban esperando, además de comprometer el tiempo de los profesionales para asistir al paciente grave ${ }^{(17)}$.

La necesidad de contener el aumento de los pacientes, principalmente en los servicios de Urgencias se hace relevante, pues son los principales responsables de la atención de usuarios graves a partir de la admisión. Además, al ser admitido, la seguridad del paciente pasa a ser responsabilidad de la institución, que debe promover el seguimiento de entrada y salida de las personas.

Se constataron 46 eventos relacionados con el cuidado, destacando la omisión de cuidado, seguido de medicación, falta de humanización, infección, caidas y fallo en el procedimiento/técnica.

La omisión de cuidados pone en riesgo la integridad física del cliente y la calidad de los servicios ofrecidos por las instituciones, contribuye a una imagen institucional negativa, genera desconfianza y eleva los costos de la asistencia ${ }^{(18)}$.

Estudio desarrollado en los Estados Unidos de América, en 36 unidades de salud, identificó como los principales y más frecuentes errores de medicación los relacionados con el horario, la omisión y la dosis equivocada ${ }^{(19)}$, evidenciando que esta realidad ocurre no solamente en los hospitales brasileños.

Estudios indican que cerca de $30 \%$ de los daños causados al paciente y a las instituciones están relacionados con los errores de medicación. Se estima que el costo anual de morbidad y mortalidad referentes a este evento sea aproximadamente de US\$76,6 billones ${ }^{(19)}$.

Los factores de riesgo asociados a la ocurrencia de estos eventos pueden estar relacionados con el individuo y con el sistema ${ }^{(20)}$.

En el escenario actual, el cambio de paradigma es necesario, pues además de garantizar la calidad del medicamento, el proceso de utilización debe seguir normas mínimas de seguridad. El abordaje sistémico de los errores de medicación puede revelar fallos del proceso, siendo posible implementar mejoras, disminuyendo así la ocurrencia de estos eventos ${ }^{(8,20)}$.

El método de análisis de causa raíz ha sido señalado como importante estrategia para la identificación de factores que contribuyen a la ocurrencia de incidentes relacionados con la medicación, además de identificar fallos en el proceso, permite comprender lo que de hecho sucede, de modo a prevenir y evitar que tengan lugar nuevos episodios ${ }^{(21)}$.

Se identificaron fallos en la humanización de la atención al paciente en las unidades de Urgencias, lo que infiere asociación con la superpoblación y el bajo número de profesionales, con la falta de sensibilidad y también con la falta de preparación para la resolución de las demandas del servicio.

En la relación entre pacientes y profesionales de salud, el profesional debe promover una asistencia humanizada a todos los pacientes. Con los progresos de la medicina y de la ciencia, el hecho de la práctica médica se ha vuelto más arriesgado y en 
muchos casos más impersonal y deshumanizado, emerge la importancia de reconocer los derechos sociales e individuales del paciente para asegurar una asistencia digna y garantizar el respeto a la vida humana. La humanización y derechos del paciente se refieren a principios normativos que orientan políticas y programas de salud y que deben ser evaluados en el juicio de la calidad de la asistencia sanitaria $^{(22)}$.

La infección relacionada con la asistencia se configura como un problema de salud pública, pudiendo ser de origen endógeno, cuando es causada por microrganismos pertenecientes a la popia flora del paciente o de origen exógeno, cuando transmitida por las manos de los profesionales de salud $u$ otras personas que entren en contacto con el paciente ${ }^{(23)}$.

La ocurrencia de infección es muy frecuente, especialmente, en ambientes que exigen compleja asistencia a la salud, así como el error de medicación y la caida. Están relacionados con la distancia de la enfermería con la práctica de gestión en salud, en el sentido de estandarizar técnicas y asegurar el seguimiento de protocolos operativos $^{(24)}$.

Estudios han evidenciado la importancia de la capacitación en el servicio, especialmente de la pedagogía crítico-reflexiva, para aumentar la adhesión de los profesionales sanitarios a estrategias que rompan la cadena de transmisión de la infección nosocomial ${ }^{(25)}$. Las acciones educativas en el servicio son consideradas más efectivas cuando se desarrollan de forma sistemática e involucrando a la mayor cantidad de profesionales posibles ${ }^{(26)}$.

La caida, especifícamente, es un evento no planeado, que puede ocasionar lesiones y perjudicar la integridad física y emocional de los pacientes. Resulta en aumento de los costos hospitalarios, aumento del tiempo de ingreso y hasta en muerte. Estudios señalan la necesidad de conocer el perfil del paciente que presenta mayor riesgo para caida a fin de posibilitar la programación de medidas preventivas y específicas de seguridad, como adecuación física del ambiente y del mobiliario hospitalario, de modo a preservar la salud de los pacientes ${ }^{(24,27)}$.

Los incidentes pueden resultar en pérdidas de naturaleza emocional, física y financiera del equipo, de la familia, de la institución y de la sociedad. Sin embargo, las consecuencias más preocupantes están relacionadas con el paciente, ya que puede agravar sus condiciones clínicas y causarle daños temporales, permanentes y hasta la muerte ${ }^{(18,24,28)}$.

El desarrollo de la cultura de seguridad, o gerencia de los riesgos a que los pacientes están expuestos y la capacitación de los profesionales de salud pueden ayudar a la adopción de buenas práticas, influyendo una actuación profesional de excelencia.

En la relación entre el paciente y profesional sanitario está implícito el principio de cuidado y, cuando ocurren errores, hay una violación de ese principio. Cabe a las instituciones de salud elaborar y establecer normas sistematizadas para el control y reducción de los incidentes. La evaluación de estos indicadores posibilita la planificación de estrategias que mejoran el sistema sanitario, trayendo beneficios al equipo y, principalmente, al paciente. 


\section{CONCLUSIÓN}

Se constató el registro de 75 ocurrencias de incidentes en la unidad investigada y se estimó prevalencia de 1,5\%. Entre los incidentes relacionados con el cuidado se destacaron aquellos referentes a la medicación y omisión de cuidado. En cuanto a la organización del servicio, se destacaron los incidentes relacionados con el fallo en las anotaciones y/o registros en la hoja de evolución del paciente. Dos eventos causaron óbito del paciente y fueron consecuencia de la previsión y provisión de materiales y de la falta de humanización.

Se constató deficiencia en los registros en cuanto a las consecuencias de los incidentes para los pacientes, profesionales e institución que, junto a la incompleta notificación, dificultan la toma de decisión y la elaboración de estrategias preventivas y de control.

De ahí la necesidad, por tanto, de estimular la construcción y uso de instrumentos de notificación, así como de incentivar el registro completo de los incidentes, ya que estos son herramientas fundamentales de la calidad, señalando lo que puede ser mejorado en los servicios sanitarios.

Este estudio indicó que las acciones preventivas de estas ocurrencias dependen, en gran parte, de la ampliación de relatos, de conductas no punitivas y, fundamentalmente, de una búsqueda crítica sobre cada fallo identificado y que precisa ser corregido, mirando a la calidad de la asistencia y promoviendo una cultura de seguridad.

\section{REFERENCIAS}

1. Padilha KG. Ocorrências iatrogênicas na prática de enfermagem. In: Cassiane SHB, Ueta J. A segurança dos pacientes na utilização da medicação. São Paulo: Artes Médicas; 2004. p. 111-21.

2. World Health Organization. World alliance for patient safety. Taxonomy. The conceptual framework for the international classification for patient safety [Internet]. Genève; 2009 [cited 2009 dez 19]. (Final Technical Report). Available from: http://www.who.int/patientsafety/taxonomy/icps_full_report.pdf.

3. Mendes W, Travassos C, Martins M, Noronha JC. Revisão dos estudos da ocorrência de eventos adversos em hospitais. Rev Bras Epidem. 2005; 8(4):393-406.

4. Gallotti RMD. Eventos adversos: o que são? Rev. Assoc. Med. Bras. 2004; 50(2):109-26.

5. Souza RB, Silva MJP, Nori A. Pronto-Socorro: uma visão sobre a interação entre profissionais de enfermagem e pacientes. Rev Gaúcha Enferm. 2007; 28(2):242-9.

6. Oliveira RC, Camargo AEB, Cassiani SHB. Estratégias para prevenção de erros na medicação no Setor de Emergência. Rev Bras Enferm. 2005; 58(4):399-404.

7. Nascimento CCP, Toffoletto MC, Gonçalves LA, Freitas WG, Padilha KG. Indicators of healthcare results: analysis of adverse events during hospital stays Rev. Latino-am. Enferm [Internet]. 2008 [cited 2013 jun 15]; 16(4):746-51. Availabe from: http://www.scielo.br/pdf/rlae/v16n4/pt 15.pdf.

8. Reason J. El error humano. Madrid: Modus Laborandi; 2009.

9. Bohomol E, Ramos LH. Erro de medicação: importância da notificação no gerenciamento da segurança do paciente. Rev Bras Enferm. 2005; 60(1):32-6.

10. Bezerra ALQ, Silva AEBC, Branquinho NCSS, Paranaguá TTB. Análise de queixas técnicas e eventos adversos notificados em um Hospital Sentinela. Rev Enferm UERJ. 2009; 17(4):467-72. 
11. Ministério da Saúde. Portaria № 529, de 1ㅇ de abril de 2013. Institui o Programa Nacional de Segurança do Paciente [Internet]. Brasília; 2013 [cited 2013 abr 11]. Available

http://bvsms.saude.gov.br/bvs/saudelegis/gm/2013/prt0529 0104 2013.html.

12. Setz VG, D'innocenzo M. Avaliação da qualidade dos registros de enfermagem no prontuário por meio da auditoria. Acta Paul Enferm. 2009;22(3):313-7.

13. Matsuda IM, Silva DMPP, Évora YDM, Coimbra JAH. Anotações/registros de enfermagem: Instrumento de comunicação para a qualidade do cuidado? Revista Eletrônica de Enfermagem [Internet]. 2006 [cited 201315 jun]; 8(3):415-21. Available from: http://www.fen.ufg.br/revista/revista8 3/v8n3a12.htm.

14. Silva JA, Grossi ACM, Haddad MCL, Marcon SS. Avaliação da qualidade das anotações de enfermagem em unidade semi-intensiva. Esc Anna Nery [Internet]. 2012 [cited 2013 jun 10]; 16(3):576-81. Available from: http://www.redalyc.org/articulo.oa?id=127723305021.

15. Silva SC, Padilha KG. Cardiopulmonary resuscitation in the intensive care unit: theoretical considerations on factors related to iatrogenic occurrences. Rev Esc Enferm USP [Internet]. 2001 [cited 2012 mai 5]; 35(4):360-5. Available from: http://www.scielo.br/pdf/reeusp/v35n4/v35n4a07.pdf.

16. Oliveira NC, Chaves LDP. Gerenciamento de recursos materiais: o papel da enfermeira de unidade de terapia intensiva. Rev Rene [Internet] 2009 [cited 2013 jul 9]; 10(4):19-27. Available from: http://www.revistarene.ufc.br/revista/index.php/revista/article/view/566/pdf.

17. Perondi MBM, Sakano TMS, Schvartsman C. Utilização de um sistema informatizado de atendimento em pronto-socorro pediátrico com sistema de escore clínico de triagem. Einstein. 2008; 6(1):31-6.

18. Santos JO, Silva AEBC, Munari DB, Miasso Al. Sentimentos de profissionais de enfermagem após a ocorrência de erros de medicação. Acta Paul Enferm. 2007; 20(4):483-8.

19. Miasso Al, Grou CR, Cassiani SHDB, Silva AEBC, Fakih FT. Medication errors: types, causes and measures taken in four brazilian hospitals. Rev Esc Enferm USP [Internet]. 2006 [cited 2013 mai 2010]; 40(4):524-32. Available from: http://www.scielo.br/scielo.php?script=sci arttext\&pid=S0080-62342006000400011.

20. Pereira CDFD, Tourinho FSV, Ribeiro JLS, Fernandes LGG, Medeiros PD, Medeiros SB. Erros de medicação: a enfermagem atuando para segurança do paciente. Nursing. 2011;14(163):650-55.

21. Teixeira TCA, Cassiani SHDB. Root cause analysis: evaluation of medication error at a university hospital. Rev Esc Enferm USP [Internet]. 2010 [cited 2013 jun 10]; 44(1):138-46. Available from: http://www.scielo.br/pdf/reeusp/v44n1/a20v44n1.pdf.

22. Vaitsman J, Andrade GRB. Satisfação e responsividade: formas de medir a qualidade e a humanização da assistência à saúde. Ciênc Saúde Coletiva. 2005; 10(3):599-613.

23. Carmagnani MIS. Segurança e Controle de Infecção. Rio de Janeiro: Reichmann e Affonso Editous; 2002.

24. Bezerra ALQ, Queiroz ES, Weber J, Paranaguá TTB. Reacciones adversas: indicadores de resultados según la percepción de las enfermeras de un hospital centinela. Enfermeria Global [Internet]. 2012 [cited 2013 jul 10]; 11(3): 198-209. Available from: http://revistas.um.es/eglobal/article/view/eglobal.11.3.155151/136281. 25. Silva EFF, Chrizostimo MM, Azevedo SL, Souza DF, Braga ALS, Lima JL. Un desafío para el controlador de infección: falta de adhesión de la enfermería a las medidas de prevención y control. Enfermeria Global [internet]. 2013 [cited 2013 jul 10]; 12(3):316-29. Available from: http://revistas.um.es/eglobal/article/view/149491/150051. 
26. Azevedo Filho FM, Martins IMS, Soares CSRS, Fazendeiro PG, Paranaguá TTB, Bezerra ALQ. Administración de medicamentos: conocimiento de los enfermeros del sector de urgencia y emergência. Enfermeria Global [Internet]. 2012 [cited 2013 jul 10]; 11(2): 54-69. Available from: http://revistas.um.es/eglobal/article/view/eglobal.11.2.142011/133851.

27. Paiva MCMS, Paiva SAR, Berti HW, Campana AO. Caracterización de las caídas de pacientes de acuerdo a las notificaciones en boletines de eventos adversos. Rev Esc Enferm USP [Internet]. 2010 [cited 2013 mar 3]; 44(1):134-8. Available from: http://www.scielo.br/scielo.php?script=sci arttext\&pid=S0080-62342010000100019.

28. Toffoletto MC, Padilha KG. Consequences of medical errors in intensive and semiintensive care units. Rev Esc Enferm USP [Internet] 2006 [cited 2013 jul 8]; 40(2):24752. Available from: http://www.ee.usp.br/reeusp/upload/pdf/245.pdf. 